\title{
Role of Ultrasound in Neurocritical Care
}

\author{
Rashmi Bhatt $^{1}$ Puneet Khanna ${ }^{2}$ \\ ${ }^{1}$ International Training Fellow, Aberdeen Royal Infirmary, Aberdeen, \\ Scotland \\ ${ }^{2}$ Department of Anaesthesiology, Pain Medicine \& Critical Care, All \\ India Institute of Medical Sciences, New Delhi, India
}

\begin{abstract}
Address for correspondence Puneet Khanna, MD, E-141, Second Floor, Greater Kailash II, New Delhi 110 048, India (e-mail: k.punit@yahoo.com).
\end{abstract}

J Neuroanaesthesiol Crit Care 2021;8:106-111.

\begin{abstract}
Keywords

- neurocritical care

- subarachnoid hemorrhage

- traumatic brain injury

- bedside ultrasonography

- transcranial doppler

- intracranial pressure

- regional blood flow

Bedside point of care ultrasound has acquired an extremely significant role in diagnosis and management of neurocritical care, just as it has in other specialties. Easy availability and increasing expertise have allowed the intensivists to use it in a wide array of situations, such as confirming clinical findings as well as for interventional and prognostic purposes. At present, the clinical applications of ultrasonography (USG) in a neurosurgical patient include estimation of elevated intracranial pressure (ICP), assessment of cerebral blood flow (CBF) and velocities, diagnosis of intracranial mass lesion and midline shifts, and examination of pupils, apart from the systemic applications. Transcranial sonography has also found use in the diagnosis of the cerebral circulatory arrest. An increasing number of clinicians are now relying on the use of ultrasound in the neurointensive care unit for neurological as well as non-neurological indications. These uses include the diagnosis of shock, respiratory failure, deep vein thrombosis and performing bedside procedures.
\end{abstract}

\section{Introduction}

Ultrasonography (USG) provides real-time bedside information with high-spatial and temporal resolution by using a variety of techniques which include two-dimensional (2D) imaging, Doppler and color-coded Doppler, and contrast-enhanced USG imaging. ${ }^{1}$ Point-of-care ultrasound has become an indispensable tool which provides a valuable supplement to clinical observations in the intensive care mode by the high acoustic impedance of the skull, and is a cornerstone in the diagnostic assessment of patients with vascular brain disease. ${ }^{2}$ At present, the important uses of ultrasonography in neurologically injured patients include assessment of changes in intracranial pressure (ICP), cerebral blood flow (CBF) and velocities, diagnosis of intracranial mass lesions and midline shifts, and pupillary examination. This narrative review aims to evaluate the available evidence of utility of ultrasound from the specific point of view of neurocritical care. This was performed by a search of available literature on Ovid, PubMed, Google Scholar and Medline, with MeSH terms like "ultrasound in neurocritical care," "transcranial Doppler" and "optic nerve sheath diameter," keeping the focus on review articles.

Published online June 7, 2020
DOI https://doi.org/ $10.1055 / \mathrm{s}-0040-1712069$ ISSN 2348-0548.

\section{Neurological Applications and Modalities}

The discussion of use of USG in neurocritical care needs special focus on its neurological applications, as the general applications have already found widespread use and acceptance in critical care units. The two main modalities available for neurocritical care are B-mode transcranial color-coded duplex (TCCD) and transcranial Doppler (TCD) sonography, apart from two-dimensional (2D) sonography. ${ }^{3}$

These modalities find an invaluable role in estimation of raised ICP, assessment of pupillary size and reaction, assessment of brain parenchyma, and diagnosis of vascular abnormalities in the neuro intensive care unit. Apart from these, it has a role in the detection of cerebral circulatory arrest, which is otherwise a clinical diagnosis. - Table 1 lists some of the conditions which can be detected or diagnosed using ultrasonography. These applications are discussed in greater detail below, outlining the usefulness as well as limitations.

\section{Assessment of Intracranial Pressure}

Raised ICP is a condition which can have devastating consequences if not diagnosed and managed early. ICP measurement can be performed by invasive as well as noninvasive

(C) 2020. Indian Society of Neuroanaesthesiology and Critical Care. This is an open access article published by Thieme under the terms of the Creative Commons Attribution-NonDerivative-NonCommercial-License, permitting copying and reproduction so long as the original work is given appropriate credit. Contents may not be used for commercial purposes, or adapted, remixed, transformed or built upon. (https://creativecommons.org/licenses/by-nc-nd/4.0/).

Thieme Medical and Scientific Publishers Pvt. Ltd. A-12, 2nd Floor, Sector 2, Noida-201301 UP, India 
Table 1 Common findings and pathologies in neurocritical care patients

\begin{tabular}{|l|l|}
\hline $\begin{array}{l}\text { Pathology/ } \\
\text { condition }\end{array}$ & Role of USG/TCD \\
\hline $\begin{array}{l}\text { Traumatic } \\
\text { brain injury }\end{array}$ & $\begin{array}{l}\text { Noninvasive estimation of ICP and CPP } \\
\text { Cerebral autoregulation } \\
\text { Compliance and cerebrovascular dynamics } \\
\text { Posttraumatic vasospasm detection } \\
\text { Evaluation of effect of therapeutic intervention } \\
\text { Assessment of pupils }\end{array}$ \\
\hline $\begin{array}{l}\text { Subarachnoid } \\
\text { hemorrhage }\end{array}$ & $\begin{array}{l}\text { Vasospasm } \\
\text { Autoregulation and cerebrovascular reactivity } \\
\text { Noninvasive estimation of ICP } \\
\text { Evaluation of effect of therapeutic intervention }\end{array}$ \\
\hline Stroke & $\begin{array}{l}\text { Diagnosis and treatment of ischemic stroke } \\
\text { Emboli monitoring } \\
\text { Cardiac evaluation: shunts }\end{array}$ \\
\hline Brain tumors & $\begin{array}{l}\text { Noninvasive intracranial pressure } \\
\text { Midline shift estimation }\end{array}$ \\
\hline Brain death & $\begin{array}{l}\text { Presence of reverberating flow, systolic spikes } \\
\text { or the disappearance of previously registered } \\
\text { Doppler flow signals } \\
\text { In conjunction with clinical diagnosis }\end{array}$ \\
\hline
\end{tabular}

Abbreviations: CCP, cerebral perfusion pressure; ICP, intracranial pressure; TCD, transcranial Doppler; USG, ultrasonography.

methods. CT scan remains a popular noninvasive diagnostic modality to confirm signs of rise in ICP and determine the need for ICP monitoring. In comparison, ocular USG to measure the optic nerve sheath diameter (ONSD) offers a bedside alternative, which is simple, noninvasive and can provide quick and reliable information about ICP, especially in a clinical emergency. ${ }^{4}$

The optic nerve is an extension of the central nervous system (CNS), enclosed by cerebral spinal fluid (CSF) and the meninges, which are collectively known as the optic nerve sheath. The optic nerve is more distensible near the eyeball, as it is only loosely attached to the dural sheath here. Due to this direct communication between the subarachnoid space and the optic nerve, the pressure changes in the brain are closely reflected in the optic nerve and its sheath. This phenomenon has been demonstrated to occur within minutes of acute changes in ICP, and thus the ONSD poses an attractive target for noninvasive ICP monitoring. ${ }^{5}$ Measurement of the ONSD can be carried out serially over a period of time, as it is easily available and poses no radiation hazard, so any changes in the status and response to treatment can also be noted.

The preferred probe to measure ONSD is a high frequency (5-10 MHz) linear probe, with the depth setting adjusted to 5 to $6 \mathrm{~cm} .^{6}$ The examination is performed in the supine position, with eyes closed, and midline position of the eyes can be maintained in awake or cooperative patients. After applying a generous amount of ultrasound gel over the closed eyelids, the transducer is placed gently on the closed eye, and adjusted to obtain an image of the

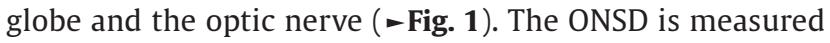
$3 \mathrm{~mm}$ posterior to the globe, with the normal value being $4.5 \mathrm{~mm}$ (-Fig. 2). A value more than $5.0 \mathrm{~mm}$ is suggestive of an ICP $>20 \mathrm{~mm} \mathrm{Hg}$ in ventilated patients. ${ }^{7}$

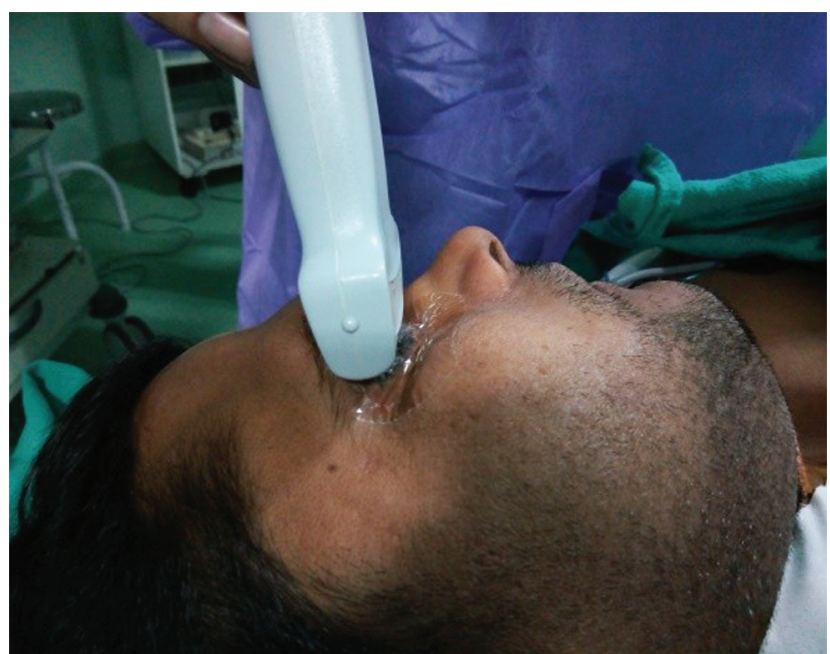

Fig. 1 Position of transducer for measurement of ONSD. OSND, optic nerve sheath diameter.

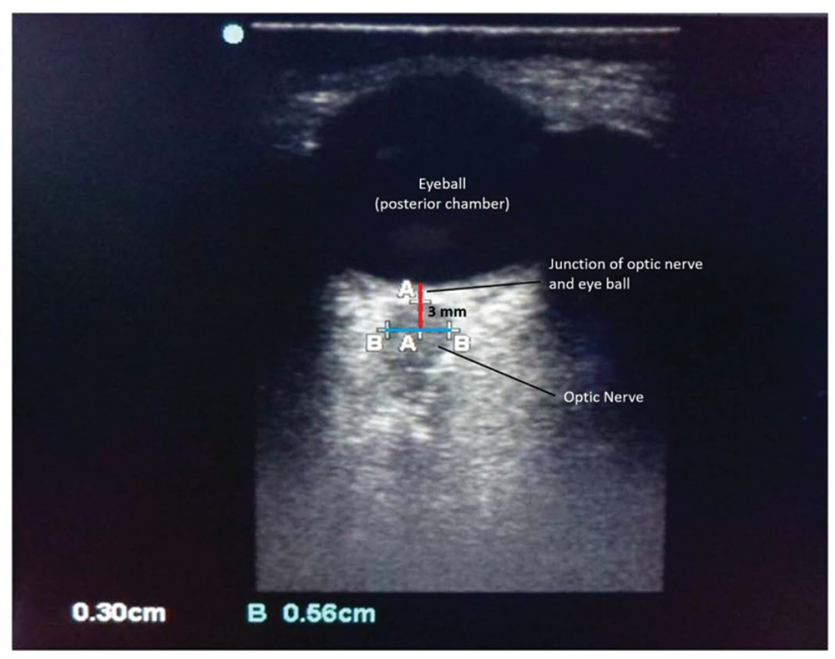

Fig. 2 Sonographic image of optic nerve and eyeball. For measurement of ONSD, a $3 \mathrm{~mm}$ line $(A-A)$ is drawn from junction of eyeball and optic nerve. At this point another line is drawn perpendicular to the first line (B-B) to measure the ONSD. OSND, optic nerve sheath diameter.

Care must be taken to avoid the use of ONSD in patients with orbital trauma, hematoma and exophthalmos. ${ }^{8}$ It may be erroneous in patients with optic neuritis, optic nerve atrophy and glaucoma, and hence better avoided. It must also be noted that its use in the pediatric population is limited, as the relationship between ONSD and raised ICP is dependent on establishing and validating threshold values above which ICP is considered elevated. These values have been less specifically established in children, due to more inter individual variations.

It has been demonstrated that combining ONSD measurement with other ultrasound modalities, like venous transcranial Doppler assessment of the straight sinus, may provide better prognostic accuracy for the detection of intracranial hypertension. ${ }^{9}$

\section{Pupillary Assessment}

USG may be a useful tool in pupillary assessment in patients with grossly swollen eyelids, trauma or soft tissue damage which prevents the eyelids from opening. The simple 
evaluation of the consensual pupillary reflex can test the integrity of the retina, optic nerve, part of the midbrain, and the oculomotor nerve. The technique described by Sargsyan et al and can be performed by either superior or inferior approach. ${ }^{10,11}$ The superior approach uses a high frequency (5-12 MHz) linear probe and the supine patient is directed to gaze downward toward the feet. On the superior aspect of the orbit, the probe is aligned with the plane of the iris to obtain the anechoic pupillary image (-Figs. 3 and $\mathbf{4}$ ). The inferior approach differs in the placement of the probe on the lower eyelid, with an upward tilt of the probe. ${ }^{12}$

Another potential application for ocular ultrasound could be evaluation of the pupillary light reflex in situations where direct observation of the pupil may be difficult or impossible, such as ocular trauma. An oblique section of the globe is obtained using the USG probe, and further tilting into a near- coronal plane allows the clinician to visualize the iris and pupil. Once this view is obtained, consensual pupillary light reflex may be evaluated by contralateral stimulation with light, using the M mode to document pupillary motion. This response may also be charted against time, allowing evaluation of finer details.

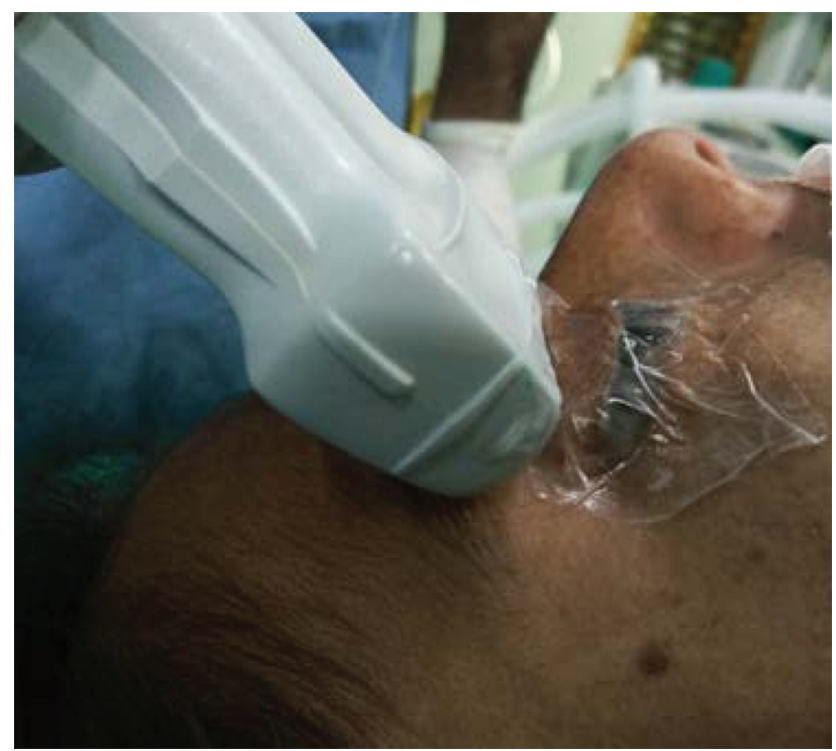

Fig. 3 Position of transducer for assessment of pupillary reflex.

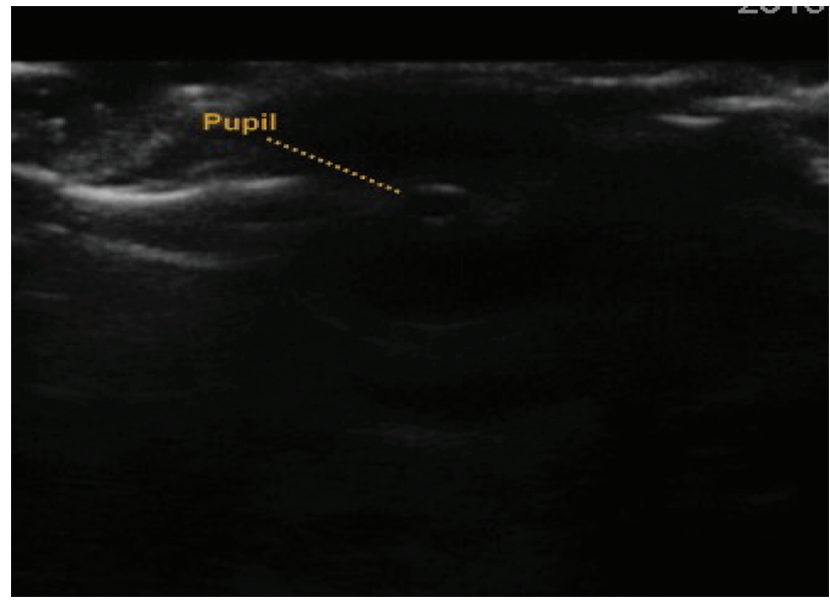

Fig. 4 B-mode image of ultrasonography showing image of pupil.

\section{Insonation of Brain Parenchyma: Transcranial B-mode Imaging}

Transcranial sonography (TCS) can be used to evaluate structural changes within the brain, which allows the identification of several structures within the brain parenchyma.

The first step while performing TCS is to accurately identify the mesencephalic brain stem. This is done by placing the phased array probe over the temporal bone window, which is parallel the orbitomeatal line. In this view, a hypoechoic butterfly-shaped structure corresponds to an axial section through the midbrain. Tilting the probe 10 to 20 degrees upward enables visualization of the ventricular plane. The landmark for orientation in this plane is the pineal gland, which is strongly hyperechoic due to calcification. The pineal gland is located adjacent to the posterior border of the third ventricle.

TCS can be used to detect as well as estimate midline shift in the cerebral parenchyma. Two techniques have been described by Seidel at $\mathrm{al}^{13}$ and Caricato et al. ${ }^{14}$ The former involves the identification of the third ventricle as a marker for the brain midline, followed by measuring the distance between the external side of the temporal bone and the third ventricle, repeating the same process on the other side. ${ }^{13}$ The midline shift can be estimated by obtaining the difference between these two measurements and halving it. This technique has been found to have a good correlation with values derived from CT. ${ }^{15}$ However, the accuracy of this technique is limited in patients with decompressive craniectomy.

The technique described by Caricato et al uses a curvilinear probe in the axial plane. ${ }^{14}$ The boundary between the two lateral ventricles is identified as the midline, followed by the falx cerebri from frontal to occipital area ( $\mathbf{- F i g}$. 5). The distance between these two landmarks can give an estimate of the midline shift (MLS).

Ultrasound assessment of MLS, correlating well with findings on $\mathrm{CT}$, is an early outcome predictor in acute stroke patients. ${ }^{16}$ An excellent correlation has also been observed between TCS and CT measurements of the width of the third ventricle and the lateral ventricles, and therefore early

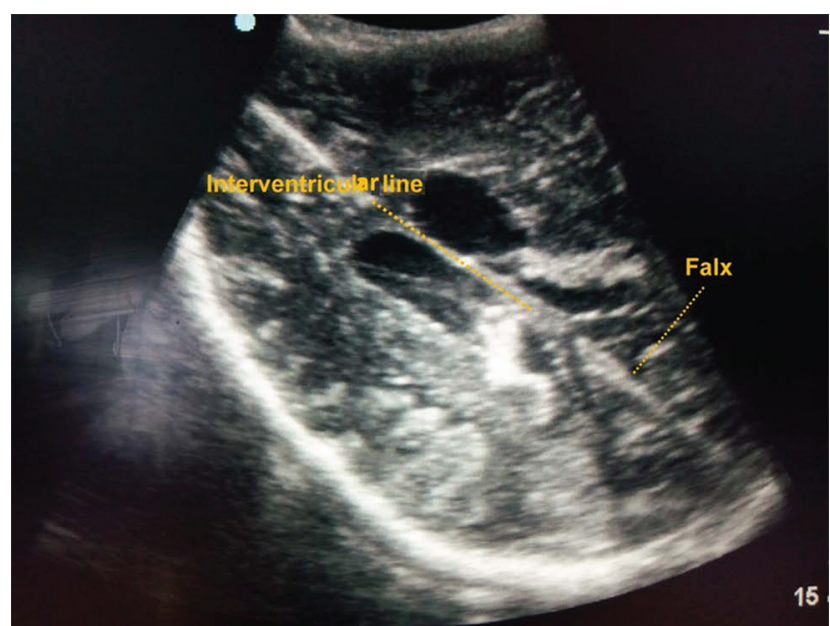

Fig. 5 Ultrasound image showing the interventricular line and falx cerebri. 
detection of development of hydrocephalus. ${ }^{13}$ Ventricular width monitoring with TCS may, in selected patients and in the hands of an experienced operator, also be useful to visualize the position of the external ventricular drain tip, especially in patients who have had a decompressive craniectomy. ${ }^{17}$

The main limiting factor for 2D USG imaging of the brain in adults is dependent on frequently inadequate acoustic windows. However, in patients with skull defects, for example with decompressive craniectomy, valuable information may be obtained about the position of the ventricular catheter, hydrocephalus and other postoperative complications like hemorrhagic lesions. ${ }^{18}$ An acoustic window can also be used as a guide for insertion or repositioning of ventricular catheter during ventriculostomy. ${ }^{19,20}$ Its use has been shown to help reduce multiple attempts and confirm correct placement, especially in patients with distorted anatomy. It has also been reported to be used to monitor intracranial bleeding and brain tumors with edema in selected patients. Research has also shown that TCS may have a role in the identification of conditions such as idiopathic Parkinson's disease, wherein patients with Parkinson's demonstrated an enlargement of the Substantia Nigra echogenic signal.

\section{Role of TCD: Diagnosis of Cerebral Vascular Abnormalities}

The detection and diagnosis of cerebral vascular abnormalities rely largely on the specific modality of transcranial Doppler (TCD). A detailed discussion which is beyond the scope of this review is not possible, but the relevant applications are discussed briefly. TCD is based on the Doppler effect, and the common insonation windows used along with their applications are summarized in - Table 2 .

Using the TCD to study cerebrovascular dynamics can facilitate clinical management of conditions such as neurocritical care pathologies, including traumatic brain injury (TBI), aneurysmal subarachnoid hemorrhage, intracranial arterial stenosis, acute ischemic stroke, and sickle cell disease, ${ }^{9}$ leading to its designation as "stethoscope for the brain."21

Among the several components of the waveform derived from TCD, diastolic flow velocity has now emerged as a clinically relevant parameter, as changes in cerebral perfusion pressure with systemic blood pressure are well-documented. ${ }^{22}$ Simultaneous monitoring with ICP and arterial blood pressure (ABP) can provide a valuable perspective in various intracranial pathologies. ${ }^{23}$ The use of TCD in patients with cerebrovascular compromise is gaining popularity, especially for the assessment of ICP and cerebral perfusion pressure (CPP), as well as detection of impairment of cerebral autoregulation. ${ }^{24,25}$ Assessment of autoregulatory capacity of the injured brain may permit more optimal blood pressure management in an individualized manner.

\section{Detection of Cerebral Circulatory Arrest: TCD}

TCD has a role in the detection of cerebral circulatory arrest, whereas the diagnosis of brain death is primarily
Table 2 Acoustic windows used for TCD examination

\begin{tabular}{|c|c|c|}
\hline $\begin{array}{l}\text { Name of acoustic } \\
\text { window }\end{array}$ & Applications/Uses & Limitations \\
\hline \multirow[t]{4}{*}{ Transtemporal } & $\begin{array}{l}\text { Thin portion of tempo- } \\
\text { ral bone }\end{array}$ & $\begin{array}{l}\text { Unsuitable in } \\
10 \% \text { patients } \\
\text { due to thick } \\
\text { bone or } \\
\text { osteoporosis }\end{array}$ \\
\hline & $\begin{array}{l}\text { Between external can- } \\
\text { thus of eye and external } \\
\text { acoustic meatus }\end{array}$ & \\
\hline & Most frequently used & \\
\hline & $\begin{array}{l}\text { Proximal segment of } \\
\text { MCA, ACA, PCA, and } \\
\text { final segment of ICA }\end{array}$ & \\
\hline \multirow[t]{3}{*}{ Transorbital } & $\begin{array}{l}\text { Ophthalmic artery, } \\
\text { carotid siphon and } \\
\text { contralateral MCA }\end{array}$ & $\begin{array}{l}\text { Avoided in } \\
\text { patients } \\
\text { with recent } \\
\text { artificial lens } \\
\text { placement }\end{array}$ \\
\hline & Probe on closed eyelid & \\
\hline & $\begin{array}{l}\text { Doppler power must be } \\
\text { reduced by } 20 \%\end{array}$ & \\
\hline \multirow[t]{2}{*}{ Suboccipital } & $\begin{array}{l}\text { Posteriorly, highest } \\
\text { point of the neck }\end{array}$ & $\begin{array}{l}\text { Inadequate } \\
\text { in } 9 \% \text { of } \\
\text { patients }\end{array}$ \\
\hline & $\begin{array}{l}\text { Suboccipital and intrac- } \\
\text { ranial portion of both } \\
\text { VA and BA }\end{array}$ & \\
\hline \multirow[t]{2}{*}{ Retromandibular } & $\begin{array}{l}\text { Extracranial approach } \\
\text { to assess distal seg- } \\
\text { ment of extracranial } \\
\text { ICA }\end{array}$ & \\
\hline & $\begin{array}{l}\text { Probe at angle of } \\
\text { mandible }\end{array}$ & \\
\hline
\end{tabular}

Abbreviations: ACA, anterior cerebral artery; BA, basilar artery; ICA, internal carotid artery; MCA, median cerebral artery; PCA, posterior cerebral artery; TCD, transcranial Doppler; VA, vertebral artery.

clinical. The main pathophysiology underlying brain death is raised ICP and a loss in cerebral autoregulation, which affects cerebral blood flow, leading to cerebral circulatory arrest. TCD criteria for the diagnosis of cerebral circulatory arrest include the presence of reverberating flow patterns, systolic spikes, or the disappearance of previously registered Doppler flow signals. ${ }^{26}$ It is desirable to record these patterns in both the anterior circulation (middle cerebral artery) and posterior circulation (basilar artery and intracranial vertebral arteries) to confirm the diagnosis. If two separate readings taken 30 minutes apart show these patterns, then circulatory arrest can be confirmed as irreversible. $^{27}$

This technique does have some limitations. In patients with subarachnoid hemorrhage, sudden changes in ICP and resulting cardiac arrest can lead to a false positive diagnosis, while it may be missed in patients with fractures, decompressive craniectomy and ventriculostomy. ${ }^{28}$ To avoid false-positive results of cerebral circulatory arrest, it is recommended that patient must be hemodynamically stable 
and conditions like hypoxemia, hypercarbia, hypothermia or metabolic derangements must be corrected. ${ }^{28}$

\section{Applications in Pediatric Neurocritical Care}

In neonates and infants, due to the presence of open fontanelles, 2D brain USG is useful to diagnose intracranial malignancies and congenital malformations. In preterm infants, cerebral hemorrhage and its different patterns (intraventricular hemorrhage and periventricular hemorrhagic infarction) can also be detected. ${ }^{29}$ Hydrocephalus is commonly diagnosed using USG, which can also be used for guided ventricular catheter placement. The role of ONSD in pediatric population has been discussed earlier in the text.

\section{Systemic Applications}

USG is useful to detect underlying cardiac conditions which may or may not be related to the neurological pathology. It is useful in the detection of atrial septal defects and patent foramen ovale, which can result in paradoxical embolism through right to left cardiopulmonary shunts, and in stroke patients presenting with acute ischemic stroke. ${ }^{30}$ Evaluation for right to left shunts can be done by injecting agitated saline into a peripheral vein, and visualizing the microembolic signals observed on the TCD. USG evaluation of cardiac anatomy and function to guide fluid management and vasoactive drug infusion, as a part of a 'goal-directed' systemic and cerebral hemodynamic management protocol, can help reduce mortality and improve outcomes in neurocritical care patients. ${ }^{31}$ Acute ventricular dysfunction is a common finding after subarachnoid hemorrhage, which can be detected on echocardiography and managed actively, to prevent further cerebral ischemia. ${ }^{32}$ USG can also be used to detect Takutsubo cardiomyopathy, which is not uncommon in patients in neurointensive units. ${ }^{33}$ It is critical to diagnose this condition early on, as the usual treatment of hypotension with vasopressors, can result in worsening of the condition. - Table 3 lists some other common cardiac indications for ultrasound in neurocritical care unit.

The role of USG in trauma patients has been well-established for several years now. Various protocols in eFAST (extended focused assessment with sonography) can help detect pneumothorax and bleeding in the pericardial, thoracic as well as peritoneal cavity. Any initial findings in the emergency room can be followed up in the critical care unit to assess need for intervention. USG can also be used as a guide to insert drains as catheters, using the Seldinger technique, thereby improving the safety of these procedures.

Point-of-care ultrasound in the neurocritical care enables the diagnosis of conditions like pleural effusion, pneumothorax, lung consolidation, pulmonary abscess, interstitialalveolar syndrome, and lung recruitment/derecruitment. It offers an easy alternative to bed side radiology, being available and repeated evaluation being feasible. It also has the advantage of being able to detect smaller amounts of fluid when compared with a chest X-ray. ${ }^{34}$

The use of the BLUE and FALLS protocols in critical care is a testament to the true versatility and usefulness of USG. ${ }^{35}$ While the BLUE protocol uses profiles for the respiratory
Table 3 Cardiac ultrasound evaluation in neurocritical patients

\begin{tabular}{|l|}
\hline $\begin{array}{l}\text { Cardiopulmonary shunts: Atrial septal defect/patent foramen } \\
\text { ovale }\end{array}$ \\
\hline Ventricular function: Global and focal \\
\hline Pericardial effusion and tamponade \\
\hline Thrombus and emboli (infective endocarditis) \\
\hline Evaluation of hypotension: volume status \\
\hline New onset arrhythmias \\
\hline Cardiomyopathy, especially Takutsubo \\
\hline
\end{tabular}

diseases like pneumonia, congestive heart failure, chronic obstructive pulmonary disease (COPD), asthma, pulmonary embolism, pneumothorax with an accuracy $>90 \%$, the FALLS-protocol, puts to use the change from A-lines to lung rockets, providing a direct biomarker of clinical volume. Its key use lies in ruling out obstructive, then cardiogenic and finally hypovolemic shock, any of which can be an accompaniment in a neurocritical patient.

Ultrasound plays an invaluable role in several procedures among critical care patients. In neurocritical care patients, vascular USG imaging has three especially relevant indications: screening for atherosclerotic plaques and flow characteristics in the aortic root and extracranial supra-aortic arteries; early diagnosis of deep vein thrombosis in the lower limbs; and guiding central venous catheterization to minimize the risk of procedure-related complications. ${ }^{36}$ The use of tracheal USG imaging before percutaneous tracheostomy effectively reduces anatomical complications, including the risk of bleeding from pretracheal vascular structures. It also contributes to decreasing the risk of puncture above the first tracheal ring, injury to surrounding structures including the posterior tracheal wall, and cranial misplacement of the tracheostomy.

\section{Safety}

Ultrasound waves interact with biological tissue through both thermal and nonthermal mechanisms, the effects of which depend on the ultrasound beam's energy and frequency, as well as the properties of the medium through which the ultrasound beam passes. ${ }^{37}$ At lower levels of exposure, such as diagnostic ultrasound, there is no established evidence of any specific harmful effect; however, limited research data are available to draw firm conclusions, especially with respect to the long-term use of ultrasound.

\section{Conclusion}

Advantages offered by USG are many: it is noninvasive, easily repeatable, available, portable, and possesses no known radiation hazard to the user as well as the patient. Its applications are also plenty, some well-established, while some are still finding acceptance and wider use. Its use in intraoperative monitoring and surgical guidance is also coming to light. As an increasing number of neurointensivists gain experience 
and skills in the use of USG, it will surely become an indispensable diagnostic and therapeutic tool.

\section{Conflict of Interest}

None declared.

\section{Acknowledgment}

The authors would like to thank Dr. Siddharth Chavali $\mathrm{MD}, \mathrm{DM}$, for his valuable feedback and contribution to the content.

\section{References}

1 Cowie BS. Focused transthoracic echocardiography in the perioperative period. Anaesth Intensive Care 2010;38(5): 823-836

2 Wartenberg KE, Schmidt JM, Mayer SA. Multimodality monitoring in neurocritical care. Crit Care Clin 2007;23(3):507-538

3 Aaslid R, Markwalder T-M, Nornes H. Noninvasive transcranial Doppler ultrasound recording of flow velocity in basal cerebral arteries. J Neurosurg 1982;57(6):769-774

4 Qayyum H, Ramlakhan S. Can ocular ultrasound predict intracranial hypertension? A pilot diagnostic accuracy evaluation in a UK emergency department. Eur J Emerg Med 2013;20(2):91-97

5 Hansen H-C, Helmke K. Validation of the optic nerve sheath response to changing cerebrospinal fluid pressure: ultrasound findings during intrathecal infusion tests. J Neurosurg 1997;87(1):34-40

6 Strumwasser A, Kwan RO, Yeung L, et al. Sonographic optic nerve sheath diameter as an estimate of intracranial pressure in adult trauma. J Surg Res 2011;170(2):265-271

7 Dubourg J, Javouhey E, Geeraerts T, Messerer M, Kassai B. Ultrasonography of optic nerve sheath diameter for detection of raised intracranial pressure: a systematic review and meta-analysis. Intensive Care Med 2011;37(7):1059-1068

8 Dubost C, Geeraerts T. Possible pitfalls when measuring the optic nerve sheath with sonography. J Surg Res 2012;173(1):e43-e44

9 Robba C, Cardim D, Tajsic T, et al. Ultrasound non-invasive measurement of intracranial pressure in neurointensive care: A prospective observational study. PLoS Med 2017;14(7):e1002356

10 Bedi DG, Gombos DS, Ng CS, Singh S. Sonography of the eye. Am J Roentgenol 2006;187(4):1061-1072

11 Sargsyan AE, Hamilton DR, Melton SL, et al. Ultrasonic evaluation of pupillary light reflex. Crit Ultrasound J 2009;1:53-57

12 Veyrac C, Couture A, Saguintaah M, Baud C. Brain ultrasonography in the premature infant. Pediatr Radiol 2006;36(7):626-635

13 Seidel G, Kaps M, Gerriets T, Hutzelmann A. Evaluation of the ventricular system in adults by transcranial duplex sonography. J Neuroimaging 1995;5(2):105-108

14 Caricato A, Mignani V, Bocci MG, et al. Usefulness of transcranial echography in patients with decompressive craniectomy: a comparison with computed tomography scan. Crit Care Med 2012;40(6):1745-1752

15 Stolz E, Gerriets T, Fiss I, Babacan SS, Seidel G, Kaps M. Comparison of transcranial color-coded duplex sonography and cranial CT measurements for determining third ventricle midline shift in space-occupying stroke. Am J Neuroradiol 1999;20(8):1567-1571

16 Gerriets T, Stolz E, Modrau B, Fiss I, Seidel G, Kaps M. Sonographic monitoring of midline shift in hemispheric infarctions. Neurology 1999;52(1):45-49
17 Robba C, Simonassi F, Ball L, Pelosi P. Transcranial color-coded duplex sonography for bedside monitoring of central nervous system infection as a consequence of decompressive craniectomy after traumatic brain injury. Intensive Care Med 2019;45(8):1143-1144

18 Behnke S, Becker G. Sonographic imaging of the brain parenchyma. Eur J Ultrasound 2002;16(1-2):73-80

19 O'Neill BR, Velez DA, Braxton EE, Whiting D, Oh MY. A survey of ventriculostomy and intracranial pressure monitor placement practices. Surg Neurol 2008;70(3):268-273

20 Strowitzki M, Komenda Y, Eymann R, Steudel WI. Accuracy of ultrasound-guided puncture of the ventricular system. Childs Nerv Syst 2008;24(1):65-69

21 Robba C, Cardim D, Sekhon M, Budohoski K, Czosnyka M. Transcranial Doppler: a stethoscope for the brain-neurocritical care use. J Neurosci Res 2018;96(4):720-730

22 Bouzat P, Almeras L, Manhes P, et al; TBI-TCD Study Investigators. Transcranial Doppler to predict neurologic outcome after mild to moderate traumatic brain injury. Anesthesiology 2016; 125(2):346-354

23 Bekker A, Wolk S, Turndorf H, Kristol D, Ritter A. Computer simulation of cerebrovascular circulation: assessment of intracranial hemodynamics during induction of anesthesia. J Clin Monit 1996;12(6):433-444

24 Martin NA, Patwardhan RV, Alexander MJ, et al. Characterization of cerebral hemodynamic phases following severe head trauma: hypoperfusion, hyperemia, and vasospasm. J Neurosurg 1997; 87(1):9-19

25 Jaggi JL, Obrist WD, Gennarelli TA, Langfitt TW. Relationship of early cerebral blood flow and metabolism to outcome in acute head injury. J Neurosurg 1990;72(2):176-182

26 Günther A. Determination of brain death: an overview with a special emphasis on new ultrasound techniques for confirmatory testing. Open Crit Care Med J 2017;4:35-43

27 Segura T, Calleja S, Irimia P, Tembl JI; Spanish Society of Neurosonology. Recommendations for the use of transcranial Doppler ultrasonography to determine the existence of cerebral circulatory arrest as diagnostic support for brain death. Rev Neurosci 2009;20(3-4):251-259

28 Hassler W, Steinmetz H, Pirschel J. Transcranial Doppler study of intracranial circulatory arrest. J Neurosurg 1989;71(2): 195-201

29 Colreavy FB, Donovan K, Lee KY, Weekes J. Transesophageal echocardiography in critically ill patients. Crit Care Med 2002; 30(5):989-996

30 Kent DM, Thaler DE. Is patent foramen ovale a modifiable risk factor for stroke recurrence? Stroke 2010;41 (10 Suppl):S26-S30

31 Robertson CS. Management of cerebral perfusion pressure after traumatic brain injury. Anesthesiology 2001;95(6):1513-1517

32 van der Bilt IA, Hasan D, Vandertop WP, et al. Impact of cardiac complications on outcome after aneurysmal subarachnoid hemorrhage: a meta-analysis. Neurology 2009;72(7):635-642

33 Park JH, Kang SJ, Song JK, et al. Left ventricular apical ballooning due to severe physical stress in patients admitted to the medical ICU. Chest 2005;128(1):296-302

34 Wong CL, Holroyd-Leduc J, Straus SE. Does this patient have a pleural effusion? JAMA 2009;301(3):309-317

35 Lichtenstein DA. BLUE-protocol and FALLS-protocol: two applications of lung ultrasound in the critically ill. Chest 2015;147(6):1659-1670

36 Bilotta F, Dei Giudici L, Lam A, Rosa G. Ultrasound-based imaging in neurocritical care patients: a review of clinical applications. Neurol Res 2013;35(2):149-158

37 Izadifar Z, Babyn P, Chapman D. Mechanical and biological effects of ultrasound: a review of present knowledge. Ultrasound Med Biol 2017;43(6):1085-1104 